\title{
Subitizing: Direct apprehension or serial processing?
}

\author{
CHARLES L. FOLK \\ NASA Ames Research Center, Moffett Field, California \\ and \\ HOWARD EGETH and HO-WAN KWAK \\ Johns Hopkins University, Baltimore, Maryland
}

\begin{abstract}
Tasks involving the judgment of numerosity in the subitizing range (1-5 elements) typically yield small but significant variations in performance as a function of the number of elements. Such a result is consistent with the existence of a serial component of numerosity processing in the subitizing range. In 1985, Sagi and Julesz reported the results of a subitizing task in which performance remained virtually constant as numerosity was varied. Three experiments are reported that explore this discrepancy. The results indicate that the discrepancy is not due to the nature of the stimuli, presentation mode, dependent measure, or level of practice used by Sagi and Julesz. All conditions showed variations in performance as a function of target numerosity. Our results are consistent with models that assume that there is a serial component to subitizing.
\end{abstract}

Suppose several discrete objects are present in the visual field. By what kind of process do we come to know how many of them there are? Broadly speaking, there would appear to be three kinds of processes available to us for ascertaining their number. First, we might directly perceive or apprehend their numerosity. Second, we might count them. Finally, if a rough approximation will suffice, we might estimate their numerosity. It is possible, of course, that the process involved would be different for different circumstances (in particular, for small and large numbers). Indeed, one of the classical findings of experimental psychology is that, in judging the number of discrete elements presented simultaneously, there is a discontinuity in performance at about six items. Kaufman, Lord, Reese, and Volkmann (1949) used the term subitizing to refer to the rapid, confident, and accurate report of six or fewer elements. The numerosity of arrays larger than this is presumably ascertained by a qualitatively different process. With long or unlimited exposure duration and an emphasis on accuracy, subjects will tend to count the stimuli; with more than six stimuli present, reaction time (RT) increases by several hundred milliseconds for each additional stimulus that is counted (for reviews see Klahr \& Wallace, 1976; Mandler \& Shebo, 1982). With an exposure duration that is too brief to permit count-

This research was supported by Grant (BNS-8420151) from the National Science Foundation and Grant (87-0180) from the Air Force Office of Scientific Research. Preparation of this article was conducted while the first author held a National Research Council-NASA ARC Research Associateship. The authors would like to thank Bert Green, Paul Mullin, Rick Pringle, Terry Sejnowski, and Ben Yuhas for helpful comments and suggestions. Reprint requests should be directed to Howard Egeth, Department of Psychology, Johns Hopkins University, Baltimore, MD 21218. ing, subjects will simply estimate numerosity; this can be accomplished with little increase in RT.

The six-item limit on subitizing has been taken to be an indication of the number of items that the mind can grasp at once (e.g., Miller, 1956), which, in turn, suggests the possibility that numerosities of up to six items can be ascertained directly (i.e., without serial counting). More detailed analysis, however, shows that even below six items the latency of subitizing judgments increases somewhat with the number of items in the display. It is common to find increases in latency in the range from 25 to $100 \mathrm{msec}$ for each additional stimulus up to about four stimuli (see Klahr \& Wallace, 1976; Mandler \& Shebo, 1982). Such a latency increase suggests that processing of numerosity even within the subitizing range may involve a serial component. Models that incorporate a serial component for determining the numerosity of even small arrays have been proposed by Klahr and Wallace (1976) and by Ullman (1984).

Contrary to the bulk of the subitizing literature, there are three reported studies that do not show a variation in performance as the number of elements in the display is increased. These studies deserve special attention in that they suggest that numerosity processing, at least in the subitizing range, can proceed in parallel without the involvement of any serial component. Atkinson, Campbell, and Francis (1976) presented from 1 to 14 identical dots or lines for $150 \mathrm{msec}$. Subjects were instructed to vocalize their numerical response and simultaneously release a response key. Latency was determined from the manual response, accuracy from the vocal one. For our present purpose, the most notable finding comes from a group of 2 subjects who were told to use the responses " 1 ," " 2 ," " 3 ," " 4 ,"' or " more than 4." For these subjects, 
mean RTs were very similar for all arrays except $N=5$, which was elevated. The subjects were evidently uncertain whether the response to 5 dots should be " 4 " or "more than 4."

Although suggestive of parallel processing, there are several potential problems with the interpretation of the Atkinson et al. (1976) data. Mandler and Shebo (1982) suggest that subjects in that study may have released the response key as soon as they realized they had a "countable" response (i.e., that the array could be held in consciousness). Mandler and Shebo (1982, Experiment 2) investigated such a judgment (respond "whether or not you could tell the total number of items presented if you were asked to do so"'), and found that mean RT increased much less (by a factor of 5) over the range of one to four items in this task than in a comparable numerosity judgment task. In the Atkinson et al. (1976) study, for all we know, the latency of the actual numerical response may have increased with display size, as those times were not measured in the condition described above. In addition, the displays in the Atkinson et al. experiment were linear, and thus numerosity was confounded with the distance between the end items. Responses may have been based on array length rather than numerosity.

Mandler and Shebo (1982, Experiment 4) also found flat functions relating RT to numerosity in the range from one to five display elements. However, the stimuli were unlike those in most previous numerosity experiments. In the typical experimental design, stimulus elements are essentially placed randomly within the visual display. (Note that the study by Atkinson et al., 1976, also violates this rule.) Mandler and Shebo, however, were specifically interested in exploring the possible role of what they called canonical patterns in subitizing. Thus, the dots for array size 2 always formed a horizontal line, for array size 3 were always arranged as an equilateral triangle (point up), for size 4 were arranged as a square, for size 5 were arranged as a square with a dot in the middle, and so forth. After some practice, the RT function was flat. This is an interesting finding, which Mandler and Shebo weave artfully into an account of the subitizing phenomenon. It appears that pattern recognition can be used to circumvent the usual, possibly serial, processes involved in subitizing.

A third study that challenges previous subitizing results and that suggests parallel processing of numerosity was reported by Sagi and Julesz (1985a, 1985b). These authors examined the nature of processing in three different tasks. In the task relevant to our present purpose, subjects had to indicate the number of targets present in a field of nontargets. The relevant details about the Sagi and Julesz experiments are as follows. Targets were mixtures of a few horizontal and vertical line segments embedded in a texture composed of diagonal line segments that were all oriented in the same direction. Subjects had to determine the number of targets irrespective of orientation (thus a stimulus containing one vertical and three horizontal targets should get the response "four'). Target numerosity was blocked; in each block of trials, the subject had to decide between two numerosities ( 1 vs. 2,2 vs. 3,3 vs. 4). Stimuli were presented briefly and followed by a pattern mask. Performance was measured in terms of accuracy at different stimulus onset asynchronies. The finding of chief interest was that the processing time needed to achieve a given level of accuracy remained constant as target number increased-a result consistent with parallel processing of target numerosity.

The research by Sagi and Julesz differs methodologically in several ways from most of the subitizing literature. For one thing, it is not common to have subjects assess numerosity in the face of distracting nontargets (although it should be said that it is not clear how this particular difference could lead to parallel processing). For another, the blocking of numerosity is unusual in this kind of research. Finally, they measured accuracy of response to masked displays with well-trained psychophysical observers instead of RT with subjects serving for just an hour or two.

The particular way in which numerosity was blocked (especially in conjunction with the use of highly practiced subjects) may have had a profound effect on the outcome of the experiment. Specifically, when numerosity is blocked, the task can become one of judging whether a stimulus is greater than or less than a specific criterion. This task can be solved by a mechanism as simple as a one-layer perceptron (see Minsky \& Papert, 1969, chap. 1). However, as is typically the case, when a unique response is required for each of several different numerosities (i.e., when the system must count inputs), a one-layer perceptron fails (see Minsky \& Papert, 1969, chap. 8). In short, the difference between Sagi and Julesz's results and most previous results may reflect a basic difference in the "computational geometry" of the blocked and mixed presentation modes. The following series of studies was an attempt to resolve the empirical discrepancy.

\section{EXPERIMENT 1}

Experiment 1 was conducted to determine if the nature of the stimuli (specifically the presence of background texture elements) used by Sagi and Julesz (1985a, 1985b) could account for the lack of a target number effect typically found in subitizing studies. Subjects indicated how many (1-4) vertical and/or horizontal line segments were present in arrays that contained diagonal texture elements (similar to those of Sagi and Julesz) in one block and in arrays that did not contain texture elements (similar to previous subitizing studies) in another, with RT as the dependent measure. If the results of Sagi and Julesz's research can be attributed to the addition of background nontargets, then one would expect RT to remain constant 
with target number when texture elements are present and to increase with target number when no texture elements are present.

\section{Method}

Subjects. The subjects were 8 students, both graduate and undergraduate, associated with Johns Hopkins University. All were paid $\$ 3.50$ for their participation.

Stimuli. Displays consisted of line segments placed in the imaginary cells of a $6 \times 6$ matrix measuring $7 \mathrm{~cm}$ vertically and horizontally. Line segments were $8 \mathrm{~mm}$ long and had a stroke width of approximately $0.7 \mathrm{~mm}$. They were drawn in black ink on white cardboard cards with a Digital LVP16 plotter. At the viewing distance of $91.5 \mathrm{~cm}$, the matrix subtended approximately $4.37^{\circ}$ of visual angle horizontally and vertically, and the line segments subtended approximately $.44^{\circ}$.

Targets, which were defined as line segments that were either horizontal or vertical, were distributed randomly among the 36 cells of the matrix, with the constraint that no two targets could appear in cells that were horizontally, vertically, or diagonally adjacent. Target number was mixed, not blocked. On any given trial, 1, 2, 3 , or 4 targets could appear with equal probability. Half of the $N=1$ trials contained a vertical target and half contained a horizontal target. On half of the 2-, 3-, and 4-target trials, the targets had the same orientation (all verticals or all horizontals were equiprobable); on the other half of the trials, one target differed in orientation from the others (horizontal and vertical discrepant targets were equiprobable). We do not consider it important that on some trials the targets were heterogeneous and on others were homogeneous. This manipulation is basically an incidental reflection of the fact that the same stimuli were used in previous research in which subjects had to indicate whether all targets were or were not identical to one another. As the effect of heterogeneous versus homogeneous targets has been found to be trivial in previous research on subitizing (see Frick, 1987; Mandler \& Shebo, 1982) as well as in our own pilot research, that factor was not considered further in the present experiments.

In the texture condition, cells that did not contain targets contained a negatively sloped diagonal line segment. As with the stimuli used by Sagi and Julesz (1985a, 1985b), the distance between targets and background diagonals was relatively small. More specifically, it was less than twice the length of the line segments. (See Sagi \& Julesz, 1987, for a discussion of the effects of interelement separation.) In the no-texture condition, cells that did not contain a target were left blank. Sample stimuli are shown in Figure 1.

Procedure. The displays were presented in an Iconix four-channel tachistoscope. A fixation point was visible at the center of the field whenever a stimulus was not being presented. The sequence of events on a trial was as follows. The experimenter manually initiated a trial. One-half second after initiation of a trial, a warning tone sounded for $250 \mathrm{msec} ; 500 \mathrm{msec}$ after the offset of the tone, the stimulus was presented until the subject made a responses at which time the fixation field reappeared.

The subject's task was to indicate vocally how many targets appeared in the display (e.g., "two"). The subject spoke into a microphone; vocal RT was measured in milliseconds from stimulus onset until a Lafayette voice-activated relay (Model 18010) stopped a Lafayette clock/counter (Model 54419-A). RT and errors were recorded by the experimenter.

Trials in the two background conditions were presented in separate blocks, and the order of the conditions was balanced across subjects. In each background condition, the subjects received 16 practice trials, followed by 128 experimental trials presented in two contiguous 64-trial blocks. Short breaks were allowed between blocks of trials.

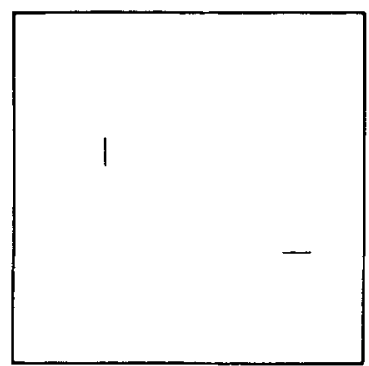

$A$

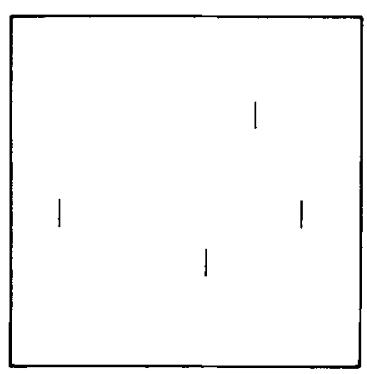

C

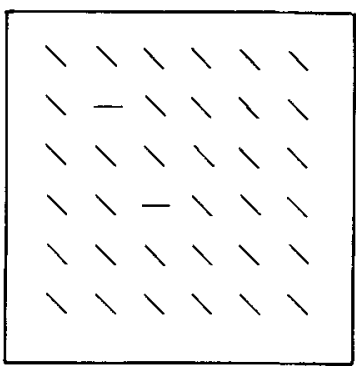

B

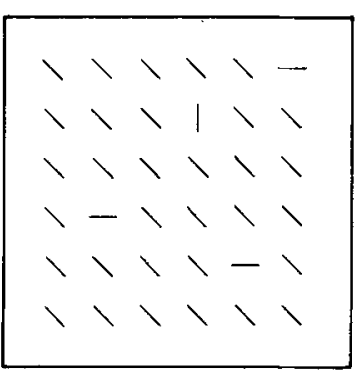

D
Figure 1. Sample stimuli used in Experiments 1, 2, and 3, illustrating texture and no-texture trial types. The correct responses to these stimuli were "two" for A and B, and "four" for C and D.

Control experiment. If spoken names of numerals are associated with different response latencies (due, for example, to differential tripping of the voice-activated relay), some correction to the counting RTs may be necessary. To find out if such a correction was necessary, the following procedure was used with each subject after he/she completed the main experiment. The subjects sat looking into a completely dark tachistoscope. At varying intervals, a bright light was flashed. The subject was instructed to say a number as quickly as he/she could with each successive flash. The flashes were counted in the following order: $1,2,3,4,1,2,3,4,1,2$, and so forth. This cycle was repeated 10 times. RT for each vocalization was measured by the same equipment described above.

\section{Results}

The control experiment yielded the following mean RTs for the words one, two, three, and four: $397,408,395$, and $405 \mathrm{msec}$, respectively. These values were not significantly different from one another $[F(3,21)<1]$. Consequently, the counting latencies were analyzed without any correction.

Mean RT for the two background conditions as a function of target number are presented in Figure 2. The data were entered into a three-way analysis of variance (ANOVA) with order (texture first vs. no texture first) as a between-subjects factor, and background (texture vs. no texture) and target number $(1,2,3$, or 4$)$ as withinsubject factors. The main effect of order was not significant, nor did it interact with any of the other factors (all ps $>.05$ ).

As suggested by Figure 2, the main effect of target number was highly significant $[F(3,18)=38.62, p<.01]$, as was the main effect of background $[F(1,6)=21.84$, 


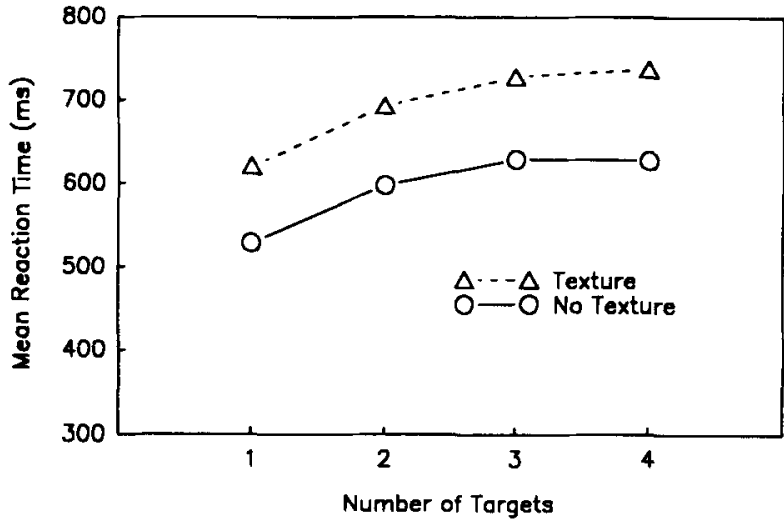

Figure 2. Mean reaction time as a function of number of targets for texture and no-texture stimuli in Experiment 1.

$p<.01]$. The interaction of target number and background was not significant $[F(3,18)=0.48, p>.05]$.

The functions for the no-texture and texture conditions were also subjected to trend analyses. The linear components of both functions were highly significant. For the notexture condizion, the slope was $32.8 \mathrm{msec}$ per target element $[F(1,7)=71.02, p<.001]$. For the texture condition, the slope was $38.3 \mathrm{msec}$ per target element $[F(1,7)$ $=21.74, p<.01]$. The negative acceleration apparent in both functions was also confirmed by significant quadratic components [for no texture, $F(1,7)=36.61, p<.001$; for texture, $F(1,7)=9.65, p<.05]$.

Errors were nearly nonexistent. No subject made more than six errors in the entire experiment. The overall error rate was just over $1 \%(.012)$, and no systematic trends were apparent. Thus, the error data were not subjected to any further analysis.

\section{Discussion}

The results of this experiment are quite clear. The addition of distractor texture elements to a counting task results in no qualitative change in performance. Although texture elements slowed performance relative to the notexture displays, both conditions showed increases in RT with the number of to-be-counted targets, a result consistent with the bulk of the subitizing literature.

One interesting result is the significant negative acceleration apparent in both background conditions. The basis for this effect is not clear. One possibility is a speedaccuracy tradeoff at higher target numbers. This is not supported by the error data, however. In fact, if anything, errors were less likely with greater target numbers. Another possibility is some form of range effect. If a subject counts four targets, he/she can be certain of the response since four is the maximum number of targets that can appear. If the subject counts fewer than four, he/she may spend extra time making sure that no other targets are present.

\section{EXPERIMENT 2}

The results of Experiment 1 suggest that it is not the nature of the stimuli themselves that led to the invariance of processing time and numerosity in the Sagi and Julesz (1985a, 1985b) experiment. In Experiment 2, we explored the possibility that their results might be due to the special "computational geometry" of the task when numerosity is blocked rather than mixed. In each block of trials, subjects had to discriminate between $N$ and $N+1$ targets. That is, in one block of trials, we presented stimuli with only one or two targets, and the subjects had to indicate whether it was one or two. In separate blocks of trials, stimuli with two or three targets or with three or four targets were presented. In each block, the subjects indicated their responses with a two-alternative forcedchoice judgment. As in Experiment 1, RT was the dependent variable of chief interest.

\section{Method}

Subjects. Twelve new subjects participated in Experiment 2 for credit. They were students at Johns Hopkins University.

Procedure. The displays used in Experiment 2 were identical to those used in Experiment1 (including target-position constraints, and the like). Half of the subjects received the texture condition first; the other half received the no-texture condition first. Each of these two conditions comprised three blocks of 64 trials corresponding to the target numbers that were to be discriminated (i.e., 1 vs. 2,2 vs. 3 , and 3 vs. 4). Within each block, the two alternative levels of numerosity were equiprobable. All possible orders of these three number-set conditions were used equally often across subjects, and the same order was used for both the texture and notexture conditions. Each block of 64 experimental trials was preceded by 16 practice trials.

The equipment and sequence of events on individual trials were identical to those used in Experiment 1, except that stimuli were exposed for $150 \mathrm{msec}$ and then immediately followed by the fixation field. As in Experiment 1, the subjects gave a vocal response to indicate the number of target elements.

\section{Results}

In Figure 3, mean RT is plotted for each number of target elements, in each number set ( 1 vs. 2,2 vs. 3 , and 3 vs. 4), and for texture and no-texture conditions. On the face of it, Figure 3 may appear to be a somewhat messy, noisy affair. In fact, however, the data are quite orderly.

The data were cast into a four-way ANOVA. The factor of order (texture first vs. no texture first) was a between-subjects factor; the other factors were all withinsubjects factors. These included texture versus no texture, number set, and, within each number set, low versus high member. (For example, in the number set 3 vs. 4, the low member is 3 and the high member is 4.)

The effect of the factor of order (texture first vs. no texture first) was not significant, and it entered into no significant interactions. It is ignored in all subsequent analyses. 


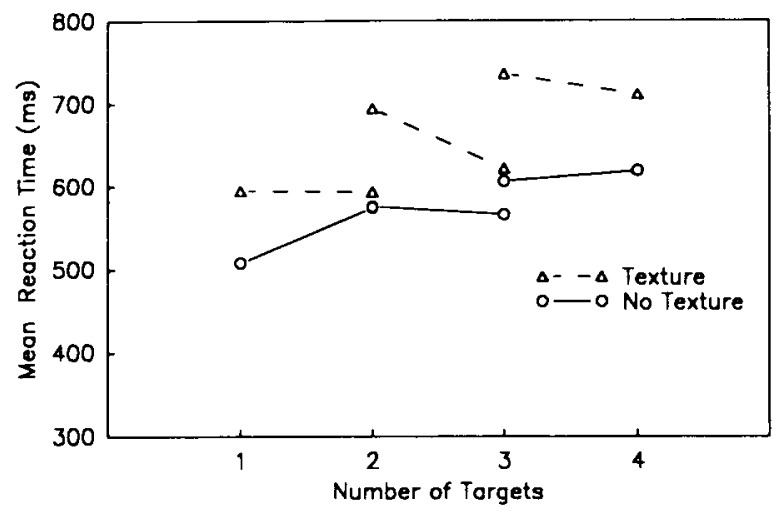

Figure 3. Mean reaction time as a function of number of targets for texture and no-texture stimuli in Experiment 2. Pairs of points connected by straight-line segments are in the same number set. Note, for example, that there are two sources of reaction times for 3-target trials-one is the 2 versus 3 discrimination and the other is the 3 versus $\mathbf{4}$ discrimination. In the former discrimination, 3 is the high member of the set; in the latter discrimination, 3 is the low member of the set.

As is evident in Figure 3, the main effect of texture versus no texture was again highly significant $[F(1,11)$ $=70.63, p<.001]$. The crucial issue in Experiment 2, however, concerns the relationship between RT and numerosity. The main effect of size of number set was highly significant $[F(2,22)=37.85, p<.001]$. Figure 4 shows the effect of number-set size collapsed across low versus high member for both texture and no-texture conditions. The slope of the function in the texture condition was $65 \mathrm{msec}$ per target element, which trend analyses showed to be significantly different from zero $[F(1,11)$ $=59.98, p<.001]$. The corresponding slope in the notexture condition was $36 \mathrm{msec}$ per target element, which is also significantly different from zero $[F(1,11)=26.99$, $p<.001]$. These two slopes differ significantly as revealed by a significant interaction of number set with texture versus no texture $[F(2,22)=4.85, p<.025]$.

Although the main effect of low versus high set member was not significant $[F(1,11)<1]$, it interacted sig-

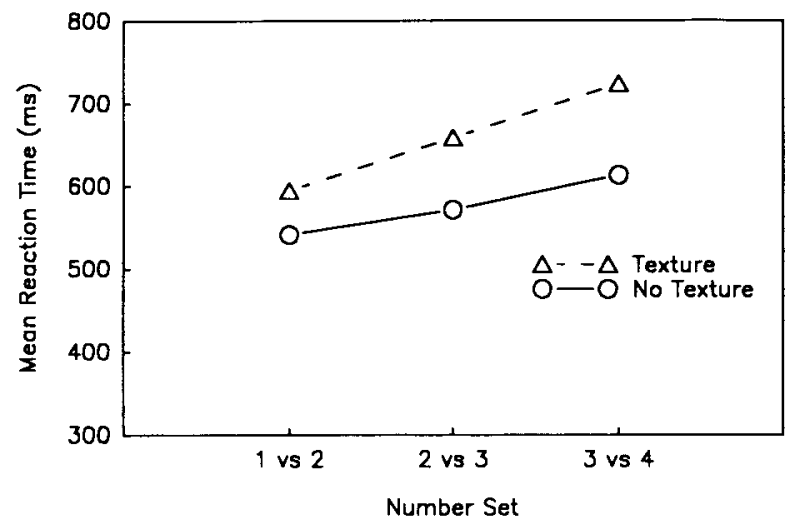

Figure 4. Mean reaction time as a function of number set for texture and no-texture conditions in Experiment 2. Data from the high and low set member are averaged for each set. nificantly with texture versus no texture $[F(1,11)=45.97$, $p<.0011$. This interaction is apparent in Figure 3: high members tend to yield longer mean RTs than the low members in the no-texture condition, while high members tend to yield shorter mean RTs than do the low members in the texture condition. The three-way interaction of number set, texture versus no texture, and low- versus high-set member was not significant $[F(2,22)=2.65$, $p<.05$ ].

Error rate overall was very low (2.4\%); some subjects made no errors in many of the conditions. Consequently, errors were not subjected to any formal statistical analysis. Informal inspection suggests, however, that there were more errors in the texture than in the no-texture condition $(3.7 \%$ vs. $1.2 \%)$. No other effect appears very prominent.

\section{Discussion}

The present results bear little resemblance to those of Sagi and Julesz (1985a, 1985b). In particular, even though we blocked values of numerosity as in their forced-choice paradigm, the time required to ascertain the number of targets in a display increased with the number of those targets. Moreover, as in Experiment 1, this effect was evident regardless of whether background texture elements were present or not.

There is an interesting peculiarity in our data that may appear to weaken our claim. Specifically, in the texture condition, high-set members tended to be responded to more quickly than low-set members. We shall argue, however, that this effect (which we had expected prior to conducting the experiment) is a predictable consequence of using a textured background. Imagine the plight of a subject in our texture condition working, say, with the 3 versus 4 number set. If the subject sees four stimuli, he/she can respond quickly. If, however, the subject sees three stimuli, he/she may not be so quick to respond as there may be another target lurking among, and hence partially "hidden" by, the background diagonals. Thus, in this two-choice discrimination task, presentation of high-set members may result in a self-terminating search process, whereas presentation of low-set members may result in an exhaustive process (or one that continues until a temporal deadline is reached). In the no-texture condition, such strategic considerations would seem to be irrelevant since there are no background elements to hide a target.

\section{EXPERIMENT 3}

It is not immediately clear to us why the results of Experiment 2 should be so different from those of Sagi and Julesz (1985a, 1985b). Among the possible explanations is the obvious fact that our method differed in detail from that of Sagi and Julesz. In particular, we measured RTs to displays that were presented for $150 \mathrm{msec}$ and were not followed by a mask, whereas Sagi and Julesz measured accuracy of response to displays that were presented 
very briefly $(5 \mathrm{msec})$ and were followed at a variable stimulus onset asynchrony (SOA) by a masking field. Additionally, our subjects served in only a single session, whereas Sagi and Julesz's subjects were trained psychophysical observers. To explore the possible relevance of these design differences, Experiment 3 was conducted. In this study, stimuli were displayed briefly and followed by a mask. Accuracy was the dependent variable of chief interest.

\section{Method}

Subjects. Six subjects served in this experiment for pay. They were students at Johns Hopkins University.

Apparatus. Displays were generated by an IBM AT computer and displayed on a Hewlett-Packard 1345A digital display module with a P31 phosphor. The display scope was mounted into one channel of an Iconix four-channel tachistoscope. A second channel in the tachistoscope provided a constant, dim background illumination. The computer collected all RT and error data.

Stimuli. The displays were similar to those used in Experiments 1 and 2 . Line segments were placed in the imaginary cells of a $8 \times 8$ matrix that measured $7.5 \mathrm{~cm}$ vertically and horizontally. The segments were $6 \mathrm{~mm}$ long and had a stroke width of approximately $0.7 \mathrm{~mm}$. At the viewing distance of $65 \mathrm{~cm}$, the matrix subtended approximately $6.86^{\circ}$ of visual angle horizontally and vertically, and the line segments subtended approximately $.53^{\circ}$.

Targets were defined as horizontal and/or vertical line segments. Target positions were distributed under the same constraints as in Experiments 1 and 2 . All displays contained negatively sloped diagonal texture elements. Displays were generated on-line, and thus no 2 subjects saw exactly the same set of displays.

Design. Each subject served in six 1-h sessions. Within each session, the subject served in three blocks of 96 trials each. Each block was devoted to a different quantitative discrimination (i.e., 1 vs. 2, 2 vs. 3, and 3 vs. 4). The proportions of specific trial types (e.g., number, orientation, and heterogeneity of targets) within each block were identical to those used in Experiment 2. The order of presentation of the three blocks was balanced across days for each subject and across subjects on each day by means of a Latin square design.

Procedure. On each trial, the subject first saw a fixation point for $1,000 \mathrm{msec}$. It then disappeared and the field remained blank for $500 \mathrm{msec}$, at which time the stimulus appeared for an amount of time that was determined separately for each subject on each day. The stimulus was followed immediately by a postmask similar to that used by Sagi and Julesz (1985a, 1985b). It consisted of randomly oriented $V s$, each $V$ with two arms meeting at a $45^{\circ}$ angle. One $\mathrm{V}$ appeared in each cell of the matrix. The postmask was shown for $500 \mathrm{msec}$ or until a response was made, whichever was less. The response was manual; the subject depressed one of two keys on a box that was on a table in front of him/her. To indicate the smaller of the two numerosities in a particular block, the subject pressed the key on the left; to indicate the larger numerosity, the subject pressed the key on the right. Accuracy was measured as the average percentage correct of the two alternatives within each number set. This was done to eliminate contamination of the data by a subjective preference toward one alternative or the other.

Exposure duration was set in a separate block of trials at the beginning of each session. The stimuli used to set duration always required the 3 versus 4 discrimination. The subjects were always started at $200 \mathrm{msec}$. A simple up-down staircase algorithm was used to set stimulus exposure duration such that the subjects were responding correctly on about $70 \%$ of the trials (Levitt, 1970). Responses on successive pairs of trials were tallied and exposure duration adjusted according to the following rules. When both trials were correct, duration was decreased by $10 \%$. When the first member of the pair was correct and the second incorrect, or when both were incorrect, duration was increased $10 \%$. Finally, if the first was incorrect and the second correct, no change was made. This procedure was carried out until five reversals in the direction of change occurred. It took less than $5 \mathrm{~min}$. Once an exposure duration was established, it was used without further change in all conditions for that subject's entire session.

\section{Results}

Exposure duration. As expected, the subjects improved with practice. Over sessions, mean exposure duration (in milliseconds) decreased as follows: 163, 131, $127,103,92,110$. Exposure duration appears to reach an asymptotic value of roughly $100 \mathrm{msec}$ after about 4 days.

Performance. The purpose of the algorithm for selecting an exposure duration was to achieve a fairly stable overall level of performance (approximately $70 \%$ correct) from session to session in the face of changes in task proficiency with practice (and the concomitant reduction in exposure duration). The method we used seemed effective despite its brevity. Mean overall percentages correct across days were: $77,73,79,75,73$, and 79 , respectively.

Figure 5 shows the main results of Experiment 3. Mean accuracy rates for each number set, day, and subject were entered into a two-way, within-subjects ANOVA, with size of number set ( 1 vs. 2, 2 vs. 3, and 3 vs. 4) and days (1 through 6) as factors. Performance decreased significantly as target numerosity increased $[F(2,10)=46.06$, $p<.01]$. Moreover, this effect was the same across the 6 days of the experiment, as target numerosity did not interact with days $[F(5,25)=0.53]$.

\section{Discussion}

Consistent with Experiments 1 and 2, and in contrast to the results of Sagi and Julesz (1985a, 1985b), performance in Experiment 3 varied directly with target numerosity. This effect was evident with accuracy as the dependent measure and at all levels of practice. The design of Experiment 3, however, was not identical to the experiments of Sagi and Julesz. In particular, in their ex-

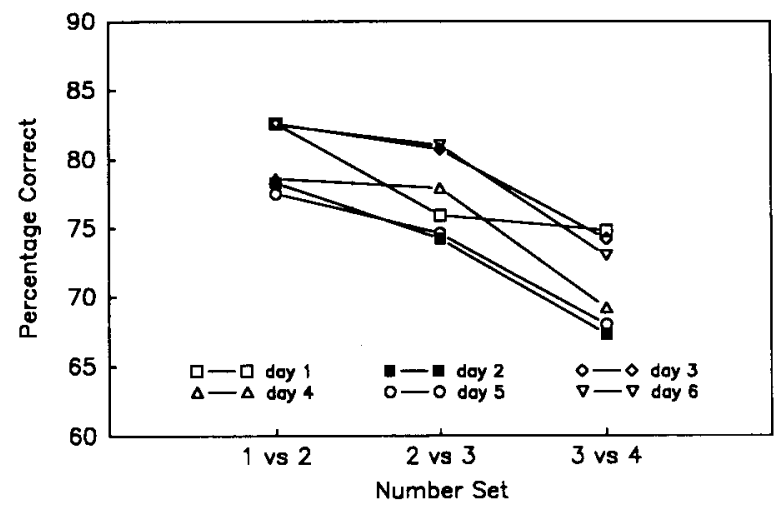

Figure 5. Accuracy (percentage correct) as a function of number set for each of six daily sessions in Experiment 3. 
periments, stimuli were displayed for $5 \mathrm{msec}$ and then followed by a blank field for a variable SOA before the 10msec presentation of the mask. The SOA was varied systematically in 50 trial blocks, thus permitting the experimenters to sweep out a complete psychometric function. The independence of performance and number of targets was illustrated in a figure (Sagi \& Julesz, 1985a, $1985 \mathrm{~b}$, Figure 2 in both cases) in which the SOA required for $95 \%$ accuracy was plotted against number of targets. The functions were essentially flat for the 3 subjects in the study. One possible problem, then, in comparing our study with those of Sagi and Julesz is that their levels of accuracy were too discrepant to make a comparison meaningful. Note, however, that their summary figure (Sagi \& Julesz, 1985a, Figure 2) merely focuses on and replots the $95 \%$ accuracy data from the psychometric functions for the individual subjects. What we can see from the full psychometric functions is that essentially the same independence of performance and number of targets holds throughout the entire range of SOAs. In particular, at the same level of performance as in Experiment 3 (i.e., at about $75 \%$ correct), the SOA required to achieve that level of performance apparently does not vary systematically with number of targets.

As an additional check on the possible effect of overall accuracy on differences among numerosity levels, we took advantage of the variability of our data around the mean with the following bit of data snooping. Recall that the staircase adjustment procedure was used to set an exposure duration for the entire session. The procedure worked well as a means for getting the subjects to operate at about the same level of overall accuracy; however, it by no means eliminated all variability among subjects, or among sessions for a given subject. We took the data for each subject and ordered his/her performance for the six sessions in terms of overall daily accuracy. These six values were then collapsed into three categories, low accuracy (the two worst sessions), medium accuracy (the two sessions intermediate in accuracy), and high accuracy (the two best sessions). Figure 6 shows accuracy as a function of size

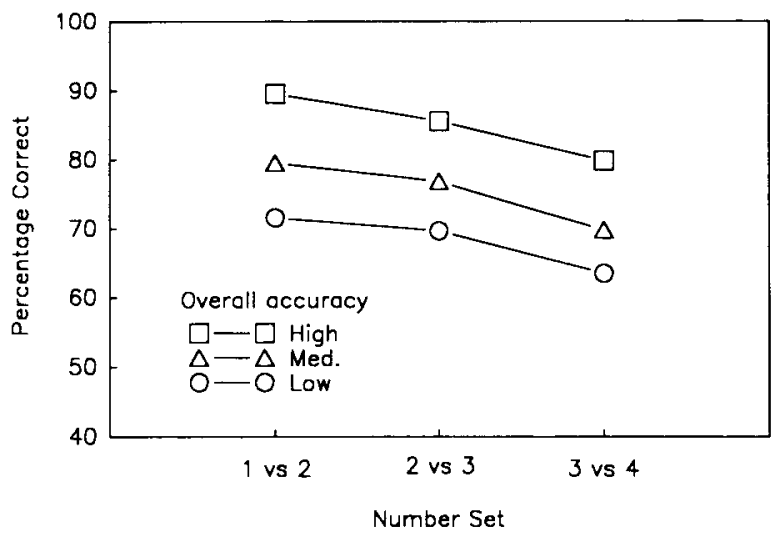

Figure 6. Accuracy (percentage correct) as a function of number set for each of three levels of overall accuracy in Experiment 3. of number set separately for the three levels of overall accuracy. These data were entered into an ANOVA in which overall accuracy level (low, medium, and high) and size of number set ( 1 vs. 2,2 vs. 3 , and 3 vs. 4) were within-subject variables. This analysis confirmed that the main effect of size of number set was significant $[F(2,10)$ $=42.48, p<.001]$. It also showed significant differences among the three levels of overall accuracy, but this is entirely unsurprising given the way the data were organized for the purpose of this analysis $[F(2,10)=27.67$, $p<.001]$. The important point of this analysis is that there was no interaction between size of number set and level of overall accuracy $[F(4,20)=0.33]$. Thus our conclusion that performance gets worse as size of number set increases seems to be unaffected by differences in overall level of performance. ${ }^{1}$

\section{GENERAL DISCUSSION}

The finding by Sagi and Julesz (1985a, 1985b) that counting, or numerosity judgment, did not take more time for larger numbers was provocative and seemed well worth investigating. Unfortunately, the results of the present series of experiments have to be considered as largely negative. Our results consistently supported the generalization that increasing numerosity leads to increases in the time required to process the stimulus. In Experiment 1, with target numerosity mixed, we found that mean RT increased for both texture and no-texture displays. In Experiment 2, the same general pattern emerged even though target numerosity was blocked. Finally, in Experiment 3, accuracy was the dependent variable, and performance was measured over 6 days of practice. Accuracy decreased as target numerosity increased regardless of the level of practice. The consistency of our results across substantial differences in methodology is quite striking. At this time, we cannot explain the discrepancy between our results and those of Sagi and Julesz. There remain unexplored differences in procedure and subject population between our research and theirs that may be responsible for the discrepancy in outcomes.

At the outset, we thought that Sagi and Julesz's result might have been due to their decision to block numerosity and require discrimination between $N$ and $N+1$ targets within each block. Even a mechanism as simple as a onelayer perceptron could solve such a problem. In more traditional studies of numerosity judgment, the number of different levels of numerosity that must be judged is much larger. A simple perceptron cannot solve such a problem. However, our own results do not suggest that the difference between the two-choice and multichoice judgment paradigms leads to any crucial qualitative difference in performance.

Sagi and Julesz concluded that knowledge of how many targets are present in a display is acquired by means of parallel processing. (This conclusion is based in part on an additional experiment reported in their 1985a paper, which we shall not discuss at this time.) It is a difficult 
matter to determine whether processing is serial or parallel in any given task (see, e.g., Townsend, 1971). We certainly would not wish to conclude that the present results necessarily indicate that processing was serial in our tasks simply because performance tended to get worse as numerosity increased. However, on the basis of our research, there seems to be little reason to reject models that assume that numerosity judgment in the subitizing range contains a serial component (e.g., Klahr \& Wallace, 1976; Ullman, 1984).

\section{REFERENCES}

Atkinson, J., Campbell, F. W., Francis, M. R. (1976). The magic number 4: A new look at visual numerosity judgments. Perception, 5, 327-334.

Fruck, R. W. (1987). The homogeneity effect in counting. Perception \& Psychophysics, 41, 8-16.

Kaufman, E. L., Lord, M. W., Reese, T. W., Volkmann, J. (1949). The discrimination of visual number. American Joumal of Psychology, 62, 498-525.

Klahr, D., Wallace, J. G. (1976). Cognitive development: An information-processing view. Hillsdale, NJ: Erlbaum.

LeviTr, H. (1970). Transformed up-down methods in psychoacoustics. Journal of the Acoustical Society of America, 49, 467-476.

Mandler, G., \& Shebo, B. J. (1982). Subitizing: An analysis of its component processes. Journal of Experimental Psychology: General, $111,1-22$.
Miller, G. A. (1956). The magical number seven, plus or minus two: Some limits on our capacity for processing information. Psychologi cal Review, 63, 81-97.

Minsky, M., PAPERT, S. (1969). Perceptrons: An introduction to com putational geometry. Cambridge, MA: MIT Press.

SAGI, D., \& JULESZ, B. (1985a). Detection versus discrimination of visua orientation. Perception, 14, 619-628.

SAGI, D., * Julesz, B. (1985b). "Where" and "what" in vision. Science, 228, 1217-1219.

SAGI, D., Julesz, B. (1987). Short-range limitation on detection o feature differences. Spatial Vision, 2, 39-49.

TOWNSEND, J. T. (1971). A note on the identifiability of parallel an serial processes. Perception \& Psychophysics, 10, 161-163.

Ullman, S. (1984). Visual routines. Cognition, 18, 97-159.

\section{NOTE}

1. We offer this posthoc analysis of the effect of overall accuracy leve with some hesitancy, as the situation does not meet the requirements of a standard fixed-effects ANOVA. Specifically, the three levels o overall accuracy were not imposed on the subjects; the subjects in : sense assigned themselves (or at least their daily performance levels) to these conditions. Among other things, this means that the three levels of overall accuracy are not necessarily quantitatively the same for different subjects. Further research with experimental control of overall accuracy level is needed to obtain definitive results.

(Manuscript received February 16, 1988; revision accepted for publication March 28, 1988.) 